\title{
Cervical varix with thrombosis diagnosed in the first trimester of pregnancy
}

\author{
Ji-Eun Park, Mi Ju Kim, Min Kyoung Kim, Hyun Mi Kim \\ Department of Obstetrics and Gynecology, Kyungpook National University Hospital, Kyungpook National University School of Medicine, Daegu, Korea
}

Cervical varix is rare and can develop due to various conditions in pregnancy. Most cases of cervical varix during pregnancy are diagnosed in the second or third trimester and are usually associated with abnormal placental location, such as placenta previa or low-lying placenta. A 23-year-old woman with cervical varix bleeding visited our institution at 8 weeks of gestation. This case report describes cervical varix bleeding that developed into a venous thrombus in the first trimester. Ultrasonography with color Doppler and magnetic resonance imaging could be helpful in diagnosis.

Keywords: Varix; Vaginal bleeding; Thrombus; Pregnancy

\section{Introduction}

Cervical varix in pregnancy is extremely rare, but may be a cause of massive vaginal bleeding. Most cases of cervical varix during pregnancy are diagnosed in the second or third trimester and are usually associated with abnormal placental location, such as placenta previa or low-lying placenta. We describe a case of first trimester vaginal bleeding caused by cervical varix that developed into a venous thrombus and also review the literature.

\section{Case report}

A 23-year-old gravida 1, para 0 patient visited the emergency department of our tertiary center because of high fever (up to $39.4^{\circ} \mathrm{C}$ ) and chills with myalgia and headache for 1 day. At the initial visit, her vital signs were stable except for the fever, and her history was unremarkable. She strongly refused possibility of pregnancy, so was not done urine pregnancy test. Computed tomography revealed no focus for a fever, but a $4.5-\mathrm{cm}$ cystic lesion was detected in the intrauterine cavity, suggesting early intrauterine pregnancy (Fig. 1A). She was referred to our obstetrics department immediately.

She had a normal menstrual cycle with subsequent amenorrhea for 8 weeks. Transvaginal ultrasonography identified a gestational sac in the uterine cavity. The fetal crown-rump length was consistent with the gestational age of 8 weeks, 6 days. The fetal heart rate was 200 per minute. The fetus, uterus, and ovaries showed no gross abnormalities. The patient had no vaginal bleeding or abnormal discharge. A speculum examination showed a very small, salmon-colored patch on the surface of anterior lip of cervix.

Intravenous antibiotics was administered because of high fever, and her fever improved. Two days after the first obstetric examination, sudden vaginal bleeding developed and persisted without abdominal pain. We repeated the physical examination and found that the cervix was severely edematous and dark red in color, suggesting ischemic change, and bled easily on contact. The vaginal bleeding was not from the cervical os, but from an edematous cervical lip. Transvaginal color Doppler ultrasonography showed a dilated venous

Received: 2017.11.01. Revised: 2018.01.23. Accepted: 2018.01.25. Corresponding author: $\mathrm{Mi}$ Ju Kim

Department of Obstetrics and Gynecology, Kyungpook National University Hospital, Kyungpook National University School of Medicine, 130 Dongdeok-ro, Jung-gu, Daegu 41944, Korea E-mail: ties1004@naver.com

https://orcid.org/0000-0001-9770-1580

Articles published in Obstet Gynecol Sci are open-access, distributed under the terms of the Creative Commons Attribution Non-Commercial License (http://creativecommons. org/licenses/by-nc/3.0/) which permits unrestricted non-commercial use, distribution, and reproduction in any medium, provided the original work is properly cited.

Copyright $\odot 2019$ Korean Society of Obstetrics and Gynecology 


\title{
Obstetrics \& Gynecology Science
}

\author{
Vol. 62, No. 1, 2019
}

plexus with abundant blood flow in the uterine cervix, suggesting a cervical varix (Fig. 1B and C).

Three days after the first episode of vaginal bleeding, no fetal heart sound was detected; hence, enhanced pelvic magnetic resonance imaging (MRI) was performed to verify the cervical varix and evaluate cervical varix status for treatment of a missed abortion. MRI revealed a diffusely dilated vascular channel with thrombus filling the surrounding uterine cervix (Fig. 1D).

Abdominal computed tomography angiography (CTA) was performed because of the possibility of massive bleeding from the cervical varix. If CTA predicts a high likelihood of massive cervical bleeding caused by a missed abortion, uterine artery embolization can be considered before treatment. CTA revealed arterial and venous cervical hypervascularity with blood supplied by the internal iliac artery (Fig. 1E). Because of her young age, we did not perform uterine artery embolization to avoid transient uterine ischemia and worsening of cervical ischemia caused by the thrombus.

Two weeks after the development of the cervical varix, dilatation and curettage were performed to treat the missed abortion. Until that day, intermittent vaginal spotting occurred. The dark-red cervical edematous lesion had improved in size and color, but the surface of the upper cervix peeled off, and was very soft and friable. After the procedure, the patient was discharged without any symptoms.

She returned to our outpatient clinic 28 days after discharge. Cervical varix was not detected on ultrasonography, but the cervical length was short (Fig. 2A). On colposcopy, the cervix had near-normal morphology, but a small red spot was observed, and the cervix seemed to be weakened, as noted for the cervix after conization (Fig. 2B). As expected, no engorged vessel was found around the cervix.
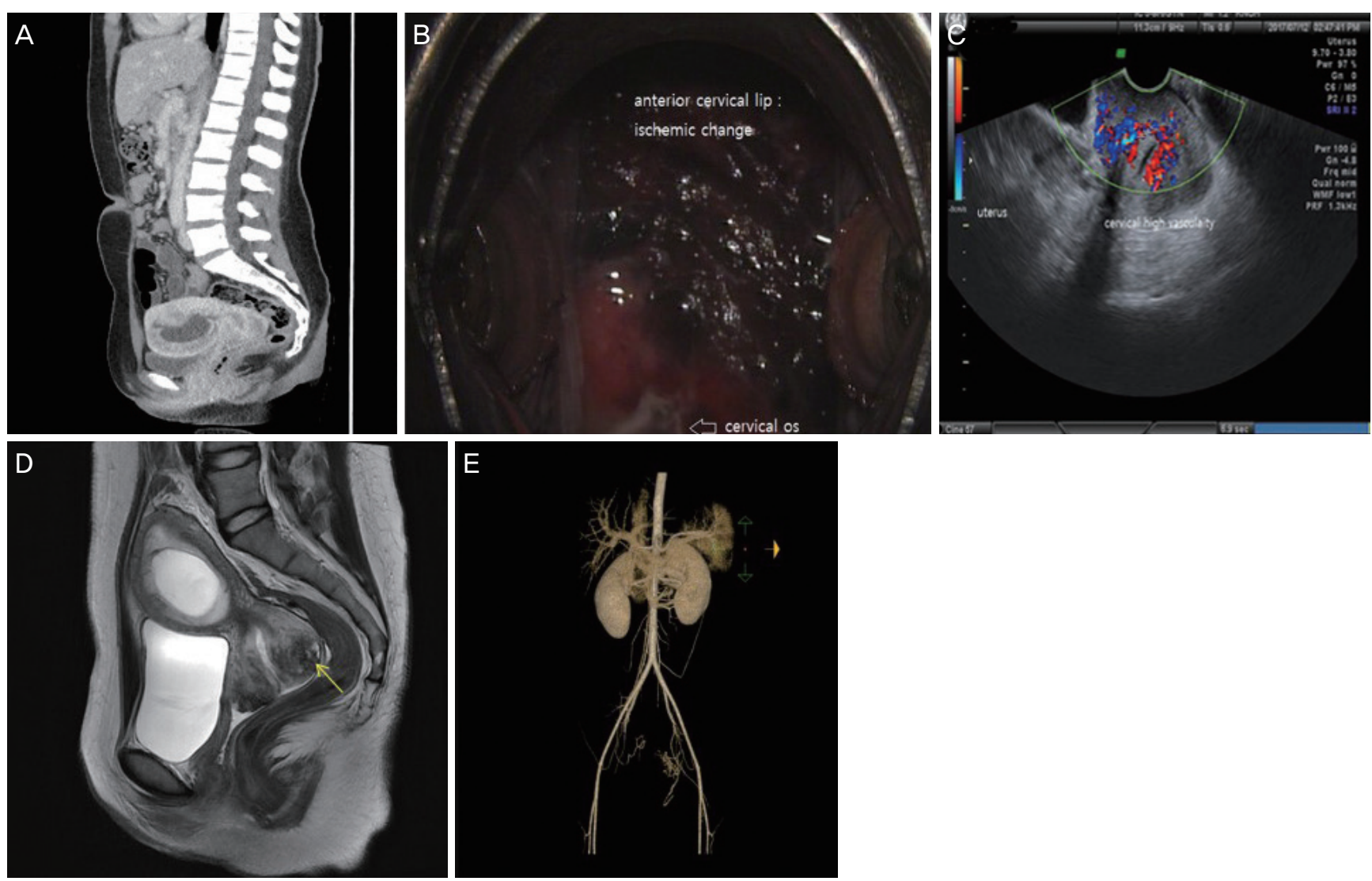

Fig. 1. (A) Computed tomography showed a cervical varix following presentation with high fever. Computed tomography revealed an intrauterine pregnancy with no other cervical abnormality. (B) The bleeding focus and cervical varix with erosion. A dark-red color change was observed in the anterior cervical lip. (C) Transvaginal ultrasonography showed cervical hypervascularity. (D) Magnetic resonance imaging showed diffuse cervical prominence with a dilated vascular channel partially filled with thrombus. (E) Computed tomography angiography showed arterial and venous hypervascularity in the cervix, which was supplied with blood from the internal iliac artery. 


\section{Obstetrics \& Gynecology Science}

Ji-Eun Park, et al. Cervical varix in pregnancy
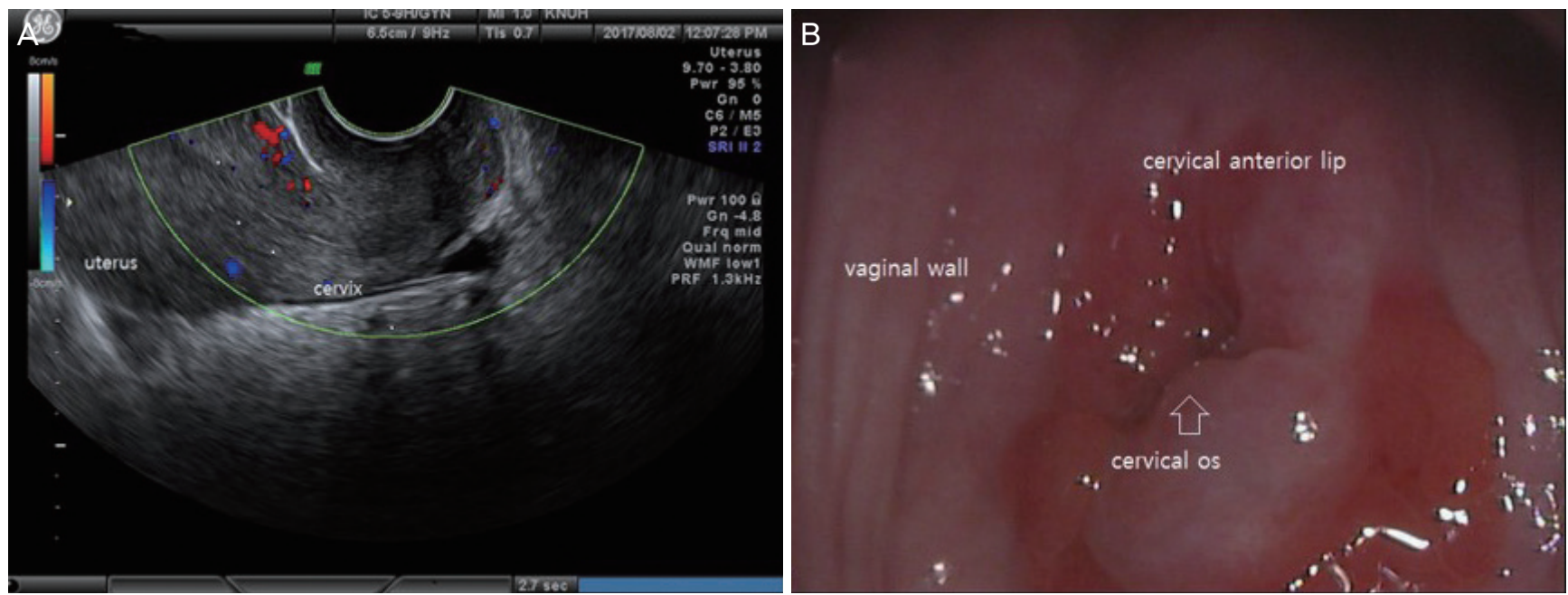

Fig. 2. Appearance at the first visit after discharge. (A) Ultrasonography showed that the cervical varix had disappeared. (B) Colposcopy showed normal morphology.

\section{Discussion}

During pregnancy, varicose veins commonly develop in the lower extremities and perineum, but rarely cause specific symptoms such as vaginal bleeding [1]. Several factors are associated with the occurrence of varicose veins in pregnancy. First, increasing plasma volume leads to venous distension [2]. Second, the inferior vena cava is compressed by increased intra-abdominal pressure during pregnancy [3]. Third, maternal hormonal changes can cause the development of varicose veins [1]. Sometimes, a cervical vascular malformation due to exposure to diethylstilbestrol can also cause a cervical varix $[4,5]$. However, uterine cervical varices are rare, especially in the first trimester of pregnancy.

Previous studies have shown that the development of cervical varix is associated with abnormal placentation. A PubMed database search found that 6 of the 11 reported cases of cervical varix were accompanied by placenta previa or a lowlying placenta [6-8]. Most patients in these cases were diagnosed in the third trimester.

Cervial varix was diagnosed during the first trimester in this case, and the varix was not related to a placental abnormality, but cervical ischemic change was induced due to thrombus.

Leibovitz et al. [9] reported 4 cases of uterine venous plexus thrombosis diagnosed in the first trimester. However, these cases were not related to cervical varices or vaginal bleeding. The causes of the cervical varix in our case were as follows:
First, the patient had a systemic infection that caused diffuse myalgia and high fever. In the septic state, the pelvic vessels are distended by endotoxins or inflammatory factors that cause thrombosis. Second, pregnancy led to expansion of the intravascular volume, predisposing the patient to thrombosis and ischemic change. Third, although we did not identify a thrombotic tendency on venous sampling, she may have had unknown thrombotic factors that may have contributed to thrombosis.

The missed abortion necessitated termination. A cervical varix can cause severe bleeding during any procedure, and prediction of the amount of bleeding is important. If massive bleeding is expected, uterine artery embolization can be considered to reduce bleeding. We performed imaging studies to determine the treatment options. MRI and abdominal CTA revealed the number of vessels present around the uterus and cervix, and if massive bleeding was expected, uterine artery embolization could reduce the amount of bleeding after the procedure. As the patient was young, uterine preservation was a priority to preserve the option of future pregnancy. There were no complications, and she was treated and discharged. Her cervix healed well, but cerclage will be needed in a future pregnancy because of possible weak cervix.

If cervical varix occurs in pregnancy without vaginal bleeding, abdominbeaal pain, or abortion, close observation is needed. However, for a missed abortion or vaginal bleeding, especially in the first trimester, an imaging analysis may be 


\section{Obstetrics \& Gynecology Science}

Vol. 62, No. 1, 2019

required for the prediction of the risk of massive bleeding during termination.

We reported a rare case of cervical varix bleeding associated with thrombus in the first trimester of pregnancy. Transvaginal color Doppler ultrasonography and MRI could be useful in diagnosing the severity of this disease.

\section{Conflict of interest}

No potential conflict of interest relevant to this article was reported.

\section{References}

1. Gabbe SG, Niebyl JR, Simpson JL. Obstetrics: normal and problem pregnancy. 4th ed. New York (NY): Elsevier; 2002.

2. Chapman AB, Abraham WT, Zamudio S, Coffin C, Merouani $A$, Young $D$, et al. Temporal relationships between hormonal and hemodynamic changes in early human pregnancy. Kidney Int 1998;54:2056-63.
3. Stansby G. Women, pregnancy, and varicose veins. Lancet 2000;355:1117-8.

4. Fleming $A D$, Anderson TL. Cervical AV malformation with in utero DES exposure. Nebr Med J 1993;78:138-9.

5. Follen MM, Fox HE, Levine RU. Cervical vascular malformation as a cause of antepartum and intrapartum bleeding in three diethylstilbestrol-exposed progeny. Am J Obstet Gynecol 1985;153:890-1.

6. Sammour RN, Gonen R, Ohel G, Leibovitz Z. Cervical varices complicated by thrombosis in pregnancy. Ultrasound Obstet Gynecol 2011;37:614-6.

7. Sükür YE, Yalçın I, Kahraman K, Söylemez F. Cervical varix complicating marginal placenta previa: a unique coexistence. J Obstet Gynaecol Res 2011;37:1515-7.

8. Kurihara Y, Tachibana D, Teramae M, Matsumoto M, Terada H, Sumi T, et al. Pregnancy complicated by cervical varix and low-lying placenta: a case report. Jpn Clin Med 2013;4:21-4.

9. Leibovitz Z, Degani S, Shapiro I, Tal J, Paz B, Levitan Z, et al. Diagnosis of pregnancy-associated uterine venous plexus thrombosis on the basis of transvaginal sonography. J Ultrasound Med 2003;22:287-93. 